\title{
Data Evacuation for Wireless Sensor Networks in Disaster Areas
}

\author{
Xiaomin Wang, Ming Liu, and Bang Liu
}

\begin{abstract}
With the increasing incidence of disasters, wireless sensor networks (WSNs) have been widely proposed to provide information for post-disaster relief operations. However, the normal assumption in pervious schemes that the deployed sensor networks can tolerate the damage caused by disasters is invalid. In reality, many significant data are blocked and eventually lost when the connectivity and coverage of WSNs are destroyed. In this paper, we demonstrate Data Evacuation (DE), a system which takes advantage of the survival time of the WSN to transmit critical data to sensor nodes in the safe area.
\end{abstract}

Index Terms-Post-disaster application, data evacuation, sensor networks.

\section{INTRODUCTION}

According to an official disaster statistic report [1], the average number of people affected by disasters is more than two hundred million per year from 1991 to 2005, and thousands of them lost their lives. Previously, wireless sensor networks have been proposed to gather useful information in disasters for relief operations. However, when a disaster strikes, the communication facilities, power units and roads will usually be destroyed, which, along with some concomitant accidents, may disrupt the normal functionalities of sensor networks. Previous researches [2]-[4] tend to overlook this possibility and result in relief solutions that are inherently impractical. Inspired by the "blackbox" solution in flight industry, we proposed Data Evacuation (DE), an original idea which utilizes the surviving time interval of sensor nodes, namely the duration in which WSNs still function after the disaster, to transmit vital data to the safe zone.

Leveraging insights from Newton's method, we propose two alternative distributed DE strategies: a gradient-based algorithm (GRAD-DE) and a gravitation-based algorithm (GRAV-DE).

The research in wireless sensor networks has rapidly grown in recent years, especially to assist humans in disaster scenarios. In [5], the Fire Bug system was composed of environmental sensors, which were used for the collection of temperature, relative humidity, atmospheric pressure. In [6], the author used sensor network to collect volcanic data. The network consists of 16 sensor nodes, each with a microphone

Manuscript received May 25, 2013; revised August 1, 2013. This work is supported by National Science Foundation under grant number 61103226, 60903158, 61170256, 61173172 and the Fundamental Research Funds for the Central Universities under grant number ZYGX2010J074 and ZYGX2011J102.

The authors are with the School of Computer Science and Engineering, University of Electronic Science and Technology of China, (e-mail: xiaomin.wangr@126.com, wing.lm@gmail.com). and seismometer, collecting seismic and acoustic data on volcanic activity. Authors in [7], [ 8] proposed to use Cellular networks to deliver message. Fujiwara et al., [9]. proposed to a data collection system with sensor networks and an access network to detect damage in a disaster and to transmit the data to an emergency operation center. Suzuki et al., [10]. introduced a system consists of seven sensor nodes and a sink node deployed on a building structure. After an earthquake, the sink node collects acceleration measurements from sensor nodes wirelessly. Analyzing these data, users can estimate damages of the structure by the earthquake. In [11], Cayirci presents a new sensor network architecture called SENDROM to manage the rescue operations after large scale disasters. Following a disaster rescue teams are assigned one mobile central node and guided to a region based on the data in the SENDROM database.

\section{DiSASTER MODEL}

Ming Liu [12] construct a simplified disaster model.

Definition 1. Define a Quaternion $\left(C_{i}, I_{i}, T_{i}, A_{i}\right)$ to represent a devastating event $E_{i}$, where $C_{i}$ is the centre point of the zone where the devastating event occurs, given by the coordinate $\left(x_{i}, y_{i}\right) ; I_{i}$ is the intensity of the devastating event, and $T_{i}$ is the attenuation coefficient of disaster propagation; $A_{i}$ is the region that the devastating event affects.

Definition 2. (Disaster) Disaster is a set of devastating events and could be denoted by $D=\left\{E_{i} \mid 0 \leq i \leq d-1, d \in \mathrm{N}\right\}$

Fig. 1 illustrates a typical intensity distribution of a disaster with four devastating events, and the intensity is collected by sensors in the affected region. The center of the four devasting events are $(15,25),(25,40),(55,85)$ and $(85,60)$. It can be seen that the intensity function has multiple sets of minimum points in its support.

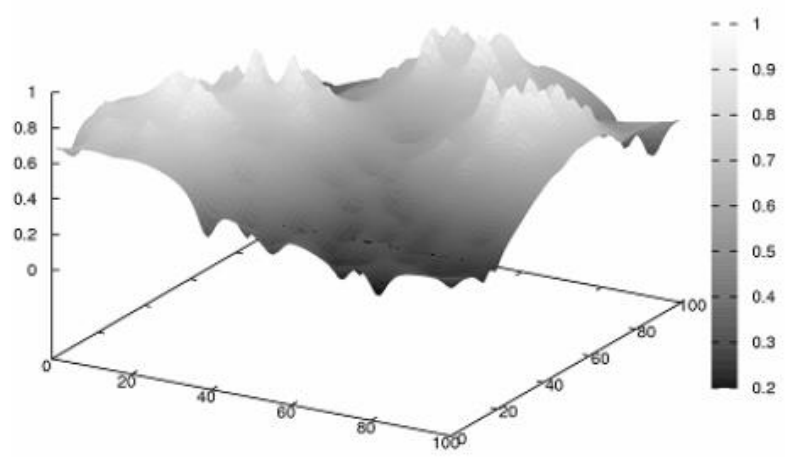

Fig. 1. Devastating event intensity distribution in disaster

We define an algorithm to classify the state of the senor node into three categories: Safe, Critical and Dangerous. 


$$
\operatorname{rank}\left(v_{i}\right)=\left\{\begin{array}{lc}
\text { Safe, } & \operatorname{intens}\left(v_{i}\right)<I_{S} \\
\text { Critical, } & I_{S} \leq \operatorname{intens}\left(v_{i}\right)<I_{D} \\
\text { Dangerous, } & I_{D} \leq \operatorname{intens}\left(v_{i}\right)
\end{array}\right.
$$

where $\operatorname{intens}\left(v_{i}\right)$ is the intensity that the node $v_{i}$ senses; $I_{s}, I_{d}$ are two thresholds which are predefined according to the disaster scene.

Fig. 2 gives a vertical view for the disaster shown in Fig. 1. In Fig. 2, most area is covered by Dangerous Zone and Critical Zone, due to the devastating event's influence, and only a small area is covered by Safe Zone.

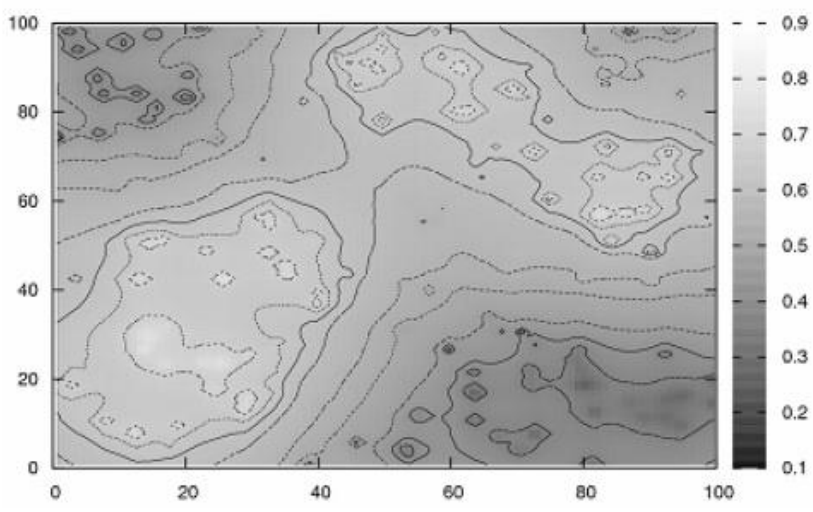

Fig. 2. Vertical view of three zones distribution

\section{DATA EVACUATION PROTOCOLS}

Ming Liu [12] present two alternatives DE protocols, each of which is a greedy algorithm seeking to optimize one design metric.

\section{A. GRAD-DE Protocol}

The GRAD-DE protocol stems from the Newton's method (gradient-based) for non-linear programming problems. One of the potential issues with Newton's method is that it could converge to local minimums. In our protocol, we allow a few steps to route the information to nodes with higher intensity, so that the critical message will not be trapped. Here is how the protocol works.

First, each sensor node obtains the intensity and the rank level of all its neighbors through a round of hello-message exchange. In the event of any disaster, a sensor node first sense the intensity of devastating event, and determine its rank level based on the predefined $I_{s}$ and $I_{d}$. After that, it will broadcast a hello message, including its sensed intensity and self-determined rank level, to all neighbors.

Second, as water always flows downwards, in the GRAD-DE protocol, each sensor node forwards the sensitive data sensed locally or received from other nodes to its neighbor with the minimum sensed intensity. Obviously, in most cases, it is reasonable to send the sensitive data to the node with lower sensed intensity because it is the most logic step toward the safe zone (also suggested by the Newton's method). In order to avoid collision and reduce the communication cost, we adopt a single-copy forwarding strategy in the design.

This simple gradient-based forwarding strategy, however, could result in data trapped in stressed zones. For example, if a disaster consists of several devastating events, the sensed intensity $\left(>I_{s}\right)$ of nodes in a certain region could be lower than the sensed intensity of any other nodes that surround this region. In this situation, the sensitive data of surrounding nodes may be forwarded to the "Highland Basin" as shown in Fig. 3, and all sensitive data in this region will be trapped.

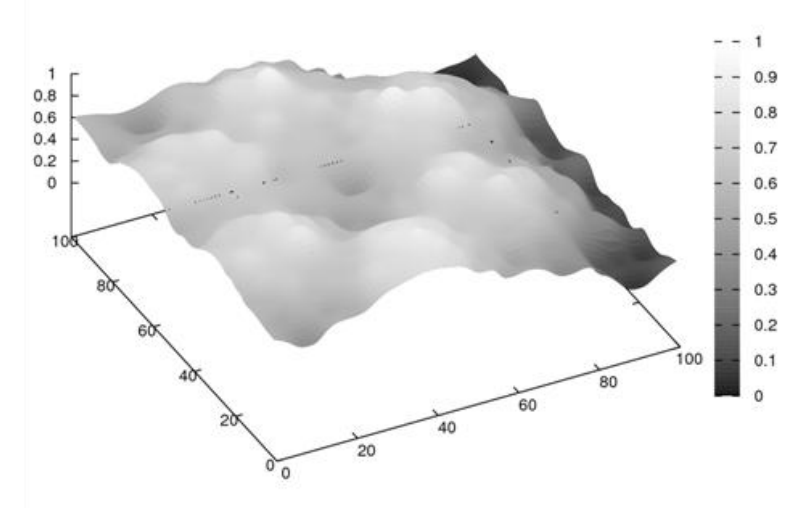

Fig. 3. "Highland Basin" phenomenon caused by the coactions of devastating events

In order to avoid this problem (equivalently, the local minimums in non-linear programming problems), the GRAD-DE algorithm consists of three correction steps as follows:

Step 1. Upon receiving all the hello messages from its neighbors, each node mark itself if its intensity is lower than that of any other neighbor, and broadcasts a warning message to prevent all its neighbors from sending sensitive data to it.

Step 2. When a node receives a warning message, if its all neighbors with smaller intensity have send warning messages to it, it will mark itself and broadcast a warning message to inform that it can not play a relay role in an effective path. Otherwise, it just drops the received warning message.

Step 3. When a node has sensitive data to forward, it will check whether it has been marked: (a) If yes, it will send the sensitive data to the unmarked neighboring node with the lowest intensity. If it can not find any unmarked neighboring node, it will send the sensitive data to the neighboring node with the highest intensity, with the hope that the data will escape from the trapped region from the trapped region as soon as possible; (b) If not, it means that this node is not located in a "Highland Basin" region, so the sensitive data can be sent to the unmarked neighboring node with the lowest intensity.

\section{B. GRAD-DE Protocol}

The GRAV-DE protocol is proposed to avoid the buffer overflow problem in the GRAD-DE protocol, which happens because each sensor node blindly forwards its data to a random safe zone. In deed, we believe that the protocol would better off if the data is forwarded to the closest safe zone with the maximum storage capacity. Such a principle is similar to Newton's theory of gravitation. Intuitively, the movement of every single sensitive message to a certain safe zone can be regarded as it is attracted by the safe zone and moves to this zone along the direction of gravitational force between them if this gravitational force is bigger than that of any other force caused by different safe zones. As a result of this parallelism, one can see a natural mapping between 
concepts in data evacuation and those in physics, as summarized in table I, where $D_{i}^{j}$ denotes the $j^{\text {th }}$ sensitive message of node $v_{i}$, and $\operatorname{space}\left(G_{j}^{s}\right)$ denotes the storage size of certain safe zone $G_{j}^{s}$.

TABLE I: CONCEPTS MAPPING

\begin{tabular}{|l|l|l|}
\hline $\begin{array}{l}\text { Concepts in } \\
\text { physics }\end{array}$ & $\begin{array}{l}\text { Concepts in data } \\
\text { evacuation }\end{array}$ & Description \\
\hline Object 1 & $\begin{array}{l}\text { Single sensitive message } \\
\text { of } v_{i}\left(D_{i}^{j}\right)\end{array}$ & $\begin{array}{l}\text { Single sensitive message } \\
\text { can been seen as an object } \\
\text { with unit mass }\end{array}$ \\
\hline Object 2 & $\begin{array}{l}\text { Space of certain safe } \\
\text { zone }\left(\operatorname{space}\left(G_{j}^{s}\right)\right)\end{array}$ & $\begin{array}{l}\text { The mass of } G_{j}^{s} \text { is the } \\
\text { storage size (messages) of } \\
\text { this safe zone }\end{array}$ \\
\hline$|\mathrm{D}|$ & Hops from $v_{i}$ to $G_{j}^{s}$ & $\begin{array}{l}\text { Distance can be expressed } \\
\text { as hops of evacuation path }\end{array}$ \\
\hline
\end{tabular}

The key for the GRAV-DE protocol is for each node in dangerous or critical zones to discover the information and distribution of safe zones, and then it can make a decision to send their sensitive data to an appropriate zone. For this object, the GRAV-DE protocol follows a three-step procedure:

1) Safe Zone Organization: in our design, each connected component or isolated node with maximum sensed intensity $<I_{s}$ can be seen as a safe zone.

2) Safe-Zone/Evacuation-Path Broadcast: After safe zones have been identified, they will advertise their existence by broadcasting announcement messages to all nodes in critical or dangerous zones.

3) Evacuation Path Decision: Nodes under stressed zones can determine the effective evacuation path for data evacuation, similar to the Newton's law of gravitation.

\section{SYSTEM DESIGN}

The hardware environment of our system is Tmote Sky wireless sensor with CC2420 antenna. The system is composed by three main parts: (1) neighbor nodes recognition, (2) devastating events triggering and propagation, (3) evacuation path decision and data transmission. Initially, all the sensor nodes will be simultaneously triggered and turn into monitoring state. Then, every node broadcasts "hello" message to find its neighbors and update the neighbors table. In (2), we randomly pick up some source nodes to be the disaster source and they will propagate the intensity of danger to their neighbors in a linearly descending order. Finally, we need to realize the DE algorithms to determine evacuation paths and transmit the data.

\section{A. Neighbor Nodes Recognition}

In order to ensure the concurrency of every part in this system, we trigger a sensor with power value 31 to send activation message to every node in this system simultaneously. When the nodes get the activation message, they will be initialized and turn to monitoring state.

The system aims to figure out evacuation paths to transmit data from critical zone to safe zone, and therefore it is a prerequisite that each node should have some knowledge about its neighbors. In this process, all the nodes broadcast "hello" messages to each other with a power value 11 . When a node receives a "hello" message, it estimates whether the
RSSI is above threshold -38 , which we predefine after numerical tests. If so, it regards the sender as a neighbor and put it into the neighbor list. Otherwise, the node considers the link between them is not well-connected and drops the "hello" message immediately.

\section{B. Devastating Events Triggering and Propagation}

When a disaster occurs in a certain place, the disaster usually only affects a limited area near the center and caused by secondary disaster often share the same zone. For simplicity, we assume there is a linear attenuation coefficient from a disaster center point to sub-regions and implement this process as following.

In the first place, every node executes a stochastic selection algorithm to determine whether it is the source node, which is denoted as disaster source $\mathrm{Si}$. In our cases, ten percent of the nodes will be picked as source nodes and marked with the highest intensity of danger. Secondly, every source node Si broadcasts a notification message, which contains an intensity value Ii, to its neighbors. Every time when a node receives a notification message, it runs a linear decrease calculation, which based on distance, to get a new value $I_{j}$ as its own intensity and sends another notification message that contains $I_{j}$ to its neighbors. If a node receives multiple notification messages, it keeps the biggest intensity and drops others. All steps proceed iteratively until every individual node gets the final intensity. When every node has its intensity, the value will be compared with threshold $h$, and the nodes with intensity bigger than $h$ will be classified in dangerous zone and the others will be in safe zone, as shown in Fig. 4.

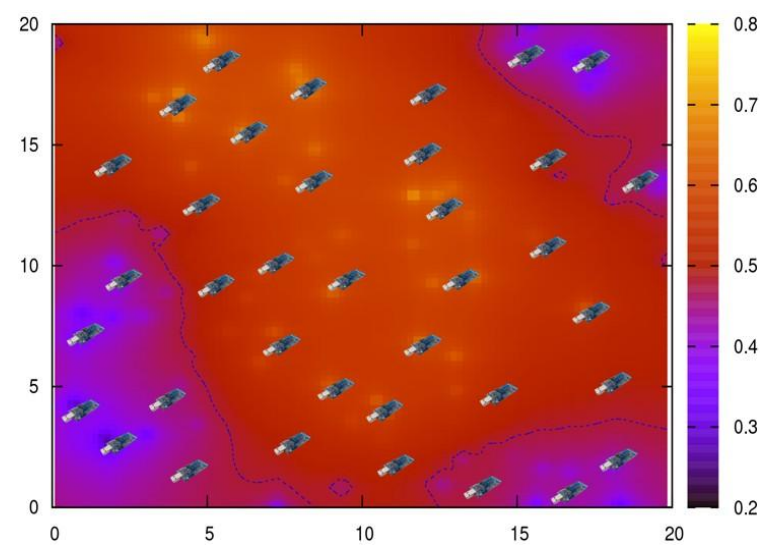

Fig. 4. Devastating events propagation

\section{Evacuation Path Decision and Data Transmission}

When every sensor node completes the first two processes, DE protocols will be executed to find data evacuation paths, including GRAD-DE protocol and GRAV-DE protocol. In GRAD-DE protocol, the only thing every node has to do is comparing its intensity with that of its neighbors and transmitting the data it senses to the neighbor with lowest intensity. GRAV-DE protocol is proposed to avoid the buffer overflow problem in GRAD-DE protocol, which happens because each sensor node blindly forwards its data to a random zone. In GRAV-DE, there are three steps: (1) safe zone organization, (2) safe-zone/evacuation-path broadcast, (3) evacuation path decision. When the evacuation paths are 
generated, sensor nodes will transmit the data from critical zone to safe zone along these paths.

\section{SimUlation}

We deployed more than forty wireless sensors in the library of UESTC to simulate the disaster scenario and investigate the effectiveness of our proposed DE algorithms.

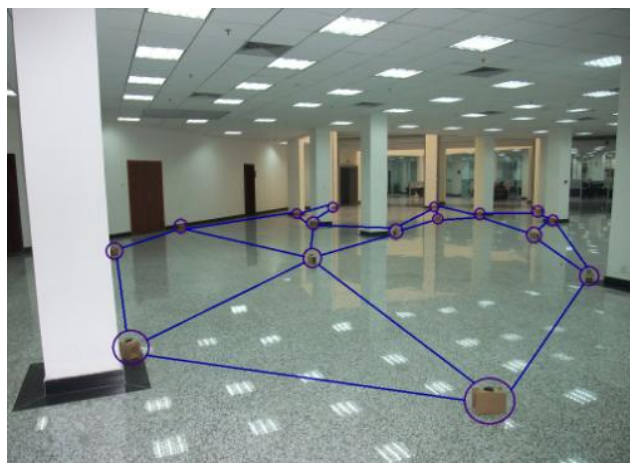

Fig. 5. Deployment in the library of UESTC

In our testbed, forty wireless sensors are randomly deployed in different positions of a $20 \mathrm{~m} \times 20 \mathrm{~m}$ grid of the library of UESTC, as shown in Fig. 5. When "devastating events" are triggered in some certain places, DE mechanism can transmit data from critical zone to safe zone within the surviving time interval of sensor nodes.

\section{A. Evacuation Ratio with Varying the Number of the Send Nodes}

We vary the number of send nodes in our simulations, with results in Fig.6.

With a rising number of sending message from 10 to 100 , GRAV-DE protocols reaches a higher evacuation ratio, which outperforms both the GRAD-DE and the flooding protocol. Flooding algorithm has a higher evacuation ratio than GRAD-DE protocol when the number of messages needed to be evacuated is very small, while drastically decreases as the number of messages increases.

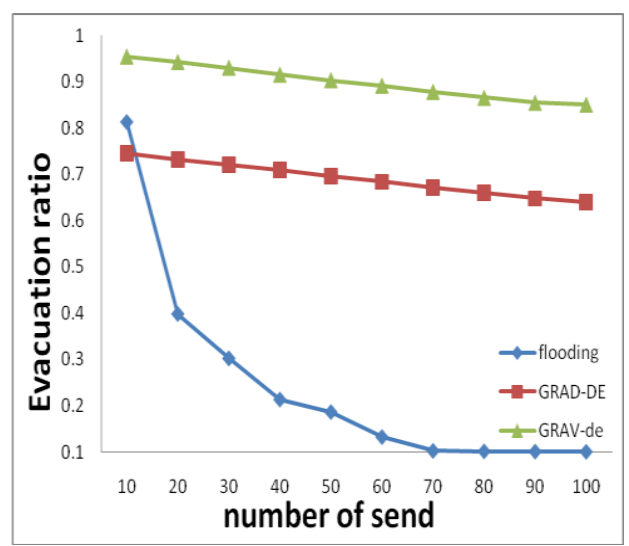

Fig. 6. Evacuation ratio with varying the number of the send nodes

\section{B. Evacuation Time with Varying the Number of the Send Nodes}

This group of experiments analyses performance of the three different protocols by varying the number of the send nodes. We vary the number of send nodes in our simulations, with results in Fig. 7.

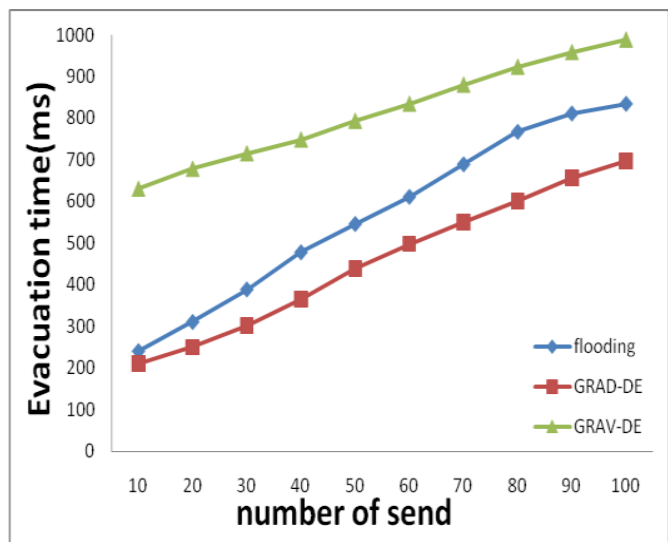

Fig. 7. Evacuation time with varying the number of the send nodes

We can easily obtain that as the number of the send nodes increases, the evacuation time of all protocols rise and GRAV-DE always attains the best performance compared with other two protocols.

\section{Evacuation Ratio with Varying Node'S Survival Time}

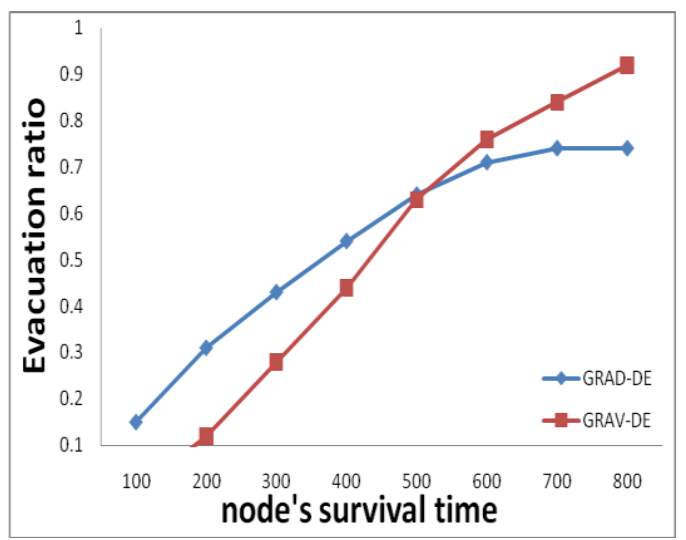

Fig. 8. Evacuation ratio with varying node's survival time

We vary the lifetime of nodes in dangerous zones from $100 \mathrm{~ms}$ to $800 \mathrm{~ms}$ in this simulation. The results are presented in Fig. 8.

As expected, with the rising survival time, the data evacuation performance of both of two schemes clearly improves.

\section{Evacuation Time with Varying Node'S Survival Time}

We can easily obtain that as the node's survival time increases, the evacuation time of two protocols rise.

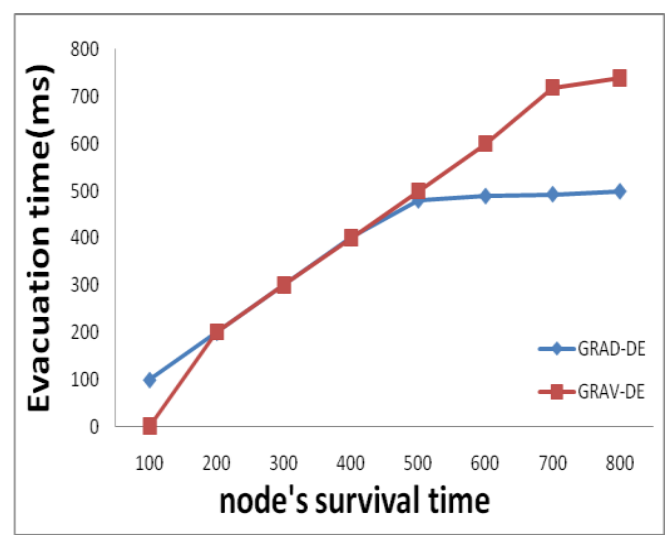

Fig. 9. Evacuation time with varying node's survival time 


\section{CONCLUSION}

This paper proposes DE strategy to transfer the critical data to safe zone when a disaster strikes. The evaluation results show the efficiency of DE algorithms.

For future research, we will compare different criteria to decide which evacuation path to take. Additionally, the associated trade-off between the evacuation time and the evacuation ratio will be evaluated.

\section{REFERENCES}

[1] Protecting the Displaced: Deepening the Responsibility to Protect. [Online]. Available: http://www.unisdr.org/disaster-statistics/pdf/isdr-disaster-statistics-im pact.pdf

[2] M. Suzuki, S. Saruwatari, N. Kurata, and H. Morkawa, "A high-density earthquake monitoring system using wireless sensor networks," in Proc. Of SenSys' 07, Sydney, 2007.

[3] H. Miura, Y. Shimazaki, N. Matusa, F. Uchio, K. Tsukada, and H. Taki, "Ubiquitous earthquake observation system using wireless sensor devices," Lecture Notes in Computer Science, vol. 5179, 2008

[4] K. Lorincz, M. Welsh, O. Marcillo, J. Johnson, M. Ruiz, and J. Lees, "Deploying a wireless sensor network on an active volcano," IEEE Internet Computing, March, 2006.

[5] J. G. Zhang and W. B. Li, "Forest fore detection system based on wireless sensor network," in Proc. The 4th IEEE Conference on Industrial Electronics and Applications, 2009, pp. 520-523

[6] G. Allen and K. Lorinez, "Deploying a wireless sensor network on an active volcano," IEEE Internet Computing. 2006, pp. 18-25
[7] Y. Yasushi and O. Toru, "Multi-hop radio access cellular concept for fourth-generation mobile communications system," IEEE PIMRC2002, 2002

[8] H. Y. Wu and C. M. Qiao, "Integrated cellular and ad hoc relaying system:icar," IEEE J-SAC, 2001, pp. 2105-2115

[9] F. Takahiro and M. Haruka, "A framework for data collection system with sensor networks in disaster circumstances," Presented at International Workshop in Wireless Adhoc Networks, 2004

[10] S. Makoto and S. Shunsuke, "A high-density earthquake monitoring system using wireless sensor networks," SenSys, 2007

[11] C. Erdal and C. Tolga, "Sendrom: sensor networks for disaster relief operations management," Wireless Networks, vol. 13, no. 3, pp. 409-423, 2003.

[12] M. Liu and H. G. Gong, "The last minute: Efficient data evacuation strategy for sensor networks in Post-Disaster applications," in Proc. the 30th IEEE International Conference on Computer Communications (IEEE INFOCOM 2011), Shanghai, 2011.

Xiaomin Wang was born in 1978. She received her Ph.D. degree from University of Electronic Science and Technology of China in Computer Architecture in 2010. She is an associate professor at UESTC. Her research interests include Wireless Sensor Networks, Delay Tolerant Mobile Sensor Networks, Vehicular Sensor Networks.

Ming Liu was born in Sichuan China in 1973. He received his PhD degree from Nanjing University in 2006. He is an associate professor at UESTC. His research interests include Wireless Sensor Networks, Vehicular Sensor Networks. 